\begin{tabular}{c} 
International Journal of Scientific World, $7(1)(2019) 1-12$ \\
International Journal of Scientific World \\
SPC \\
Website: $w$ ww.sciencepubco.com/index.php/IJSW \\
Research paper \\
\hline
\end{tabular}

\title{
Historical survey on metaheuristics algorithms
}

\author{
Saman M. Almufti * \\ Department of Computer Science and Information Technology, College of Computer Science \& Information Technology, \\ Nawroz University, Duhok, Iraq \\ *Corresponding author E-mail: Saman.Almofty@gmail.com
}

\begin{abstract}
Metaheuristic algorithms have been an interesting and widely used area for scientists, researchers and academicians because of their specific and significant characteristics and capabilities in solving optimization problems. Metaheuristic algorithms are developed base on inspiration of some real world phenomenon in nature or on the behavior of living being (animal, insects, organic living beings). On the past many metaheuristic algorithms have been introduced and applied on various problems of various domains including real world optimization problems. This paper is aimed to provide a historical Survey on metaheuristic algorithms, it will provide a list of metaheuristic based algorithms ordered according to the foundation year, with the name of Authors and the algorithm abbreviations.
\end{abstract}

Keywords: Metaheuristic; Swarm Intelligence; History; Natural Inspired Algorithm; Optimization Algorithms; Classifications

\section{Introduction}

Generally the complexity of the real life problems are in increasing in a manner that it become Difficult for the traditional mathematical programming methods to solve and optimize them. Most of the real-life optimizations problems are nonlinear, complex, multimodal, and they have a incompatible objectives functions in which the process of obtaining an optimal or even near-optimal solutions is a very difficult task, even for a single easy and linear objective functions, sometimes, an optimal solutions may not exists at all, generally, there is no guarantee of getting an optimal solution for real-life problems [1] [2].

Over the past years, Metaheuristics Optimization Algorithms become a very active area of researches and one of the most well known high-level procedure designed for generating, selecting or finding a heuristic that optimize solutions and provide a sufficiently better, improved and fittest solutions to a given objective functions for a real-life optimization problem [3] [4].

In this paper we will present a number of metaheuristics algorithm according to the year of its appearance.

\section{Metaheuristic algorithms}

In the past the methods that have a stochastic mechanisms often called heuristic algorithm, nowadays in the recent studies it refer to as metaheuristics which is a combination of two words Meta and Heuristic, were the world "heuristic" means finding or discovering a goal by trial and error, and the world "meta" means a beyond or higher level, that means a metaheuristics generally refers a "higher level of heuristics" [5]. generally metaheuristic algorithms represent as a "master strategy that guides and modifies other heuristics to produce solutions beyond those that are normally generated in a quest for local optimality" [6]. Those algorithms use a certain adjustment of randomization and local search. A good solutions for difficult optimization problems can be found in a reasonable time, but in general there is no guarantee of finding optimal solutions[1 - 4].

In the fields of computer science, mathematical optimizations and engineering's, the term "metaheuristic" represents a higher-level procedure or heuristic designed to search, find, generate, or select a heuristics, that may provide a good solution to an optimization problem, especially for the large problems such as (NP-hard problem) or incase of limit, incomplete or imperfect information[1]. Metaheuristics consists of a set of solutions which is too large to be completely sampled. Metaheuristics may make few assumptions about the optimization problem being solved, and so they may be usable for a variety of problems. [199].

Compering with optimization algorithms or iterative methods, metaheuristics do not guarantee that the best solution a globally optimal solution can be found on some class of problems [3]. Many metaheuristics implement some form of stochastic optimization, so that the solution found is dependent on the set of random variables generated [2]. In combinatorial optimization, by searching over a large set of feasible solutions, metaheuristics can often find good solutions with less computational effort than optimization algorithms, iterative methods, or simple heuristics. As such, they are useful approaches for optimization problems [2].

Most literature on metaheuristics is experimental in nature, describing empirical results based on computer experiments with the algorithms. But some formal theoretical results are also available, often on convergence and the possibility of finding the global optimum. Many metaheuristic methods have been published with claims of novelty and practical efficacy. While the field also features high-quality 
research, many of the publications have been of poor quality; flaws include vagueness, lack of conceptual elaboration, poor experiments, and ignorance of previous literature.

These are properties that characterize most metaheuristics:

- Metaheuristics are strategies that guide the search process.

- The goal is to efficiently explore the search space in order to find near-optimal solutions.

- Techniques which constitute metaheuristic algorithms range from simple local search procedures to complex learning processes.

- Metaheuristic algorithms are approximate and usually non-deterministic.

- Metaheuristics are not problem-specific.

\section{Metaheuristic algorithms major components:}

Generally metaheuristic algorithms contains two major components:

- Intensification

- Diversification

Diversifications means generating a diverse solutions so as to explore the search-space on a global scale, and the Intensification means focusing the search in a local-region that significantly believed that a current-good solution may be found in this region [7].

Balancing between those two components in the process of selecting the best-solutions improves the quality of algorithm convergence and ensures that solutions will converge to the optimum [7].

\section{Metaheuristic algorithms classifications}

Metaheuristics are broadly classified into two categories (single solution and population based) algorithms. Single solution based algorithms are those in which a solution is randomly generated and improved until the optimum result is obtained, whereas population based algorithms are those in which a set of solutions are randomly generated in a given search space and solution values are updated during iterations until the best solution is generated [2], figure 1 shows the metaheuristic classifications.

However, single solution based algorithms may trap into local optima which may prevent us to find global optimum as it reforms only one solution, which is randomly generated for a given problem. On the other hand, population based algorithms have an inherent ability to escape local optima [2]. Due to this, nowadays, population based algorithms have gained the attention of multitudinous researchers.

The categorization of population based algorithms is done on the basis of theory of evolutionary algorithms [201], physics laws based algorithms, swarm intelligence of particles, and biological behavior of bio-inspired algorithms. Evolutionary algorithms are inspired by the evolutionary processes such as reproduction, mutation, recombination, and selection. These algorithms are based on the survival fitness of candidate in a population (i.e., a set of solutions) for a given environment. The physics law based algorithms are inspired by physical processes according to some physics rules such as gravitational force, electromagnetic force, inertia force, heating and cooling of materials. Swarm intelligence based algorithms are inspired by the collective intelligence of swarms[8].

Some of the most popular evolutionary algorithms are Genetic Algorithms (GA) [6] [203], Evolution Strategy (ES) [7], Differential Evolution (DE) [8] [202], and Biogeography-Based Optimizer (BBO) [9].

A well-known algorithm of swarm intelligence technique is Particle Swarm Optimization (PSO) [10,11] [203], Elephant Herding Optimization (EHO) [202]. PSO is inspired by the social behavior of fish schooling or bird flocking. Each particle can move around the search space and update its current position with respect to the global best solution.

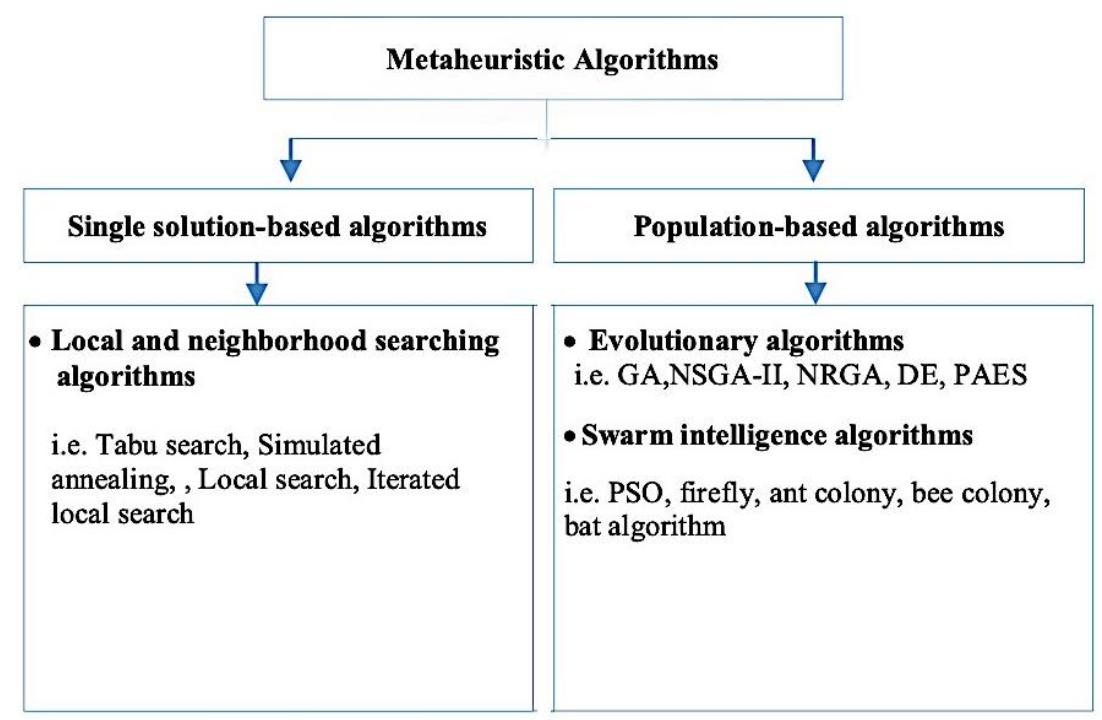

Fig. 1: Metaheuristic Classifications.

\subsection{Evolutionary algorithms (EA)}

Evolutionary algorithms (EAs) are the most well known, traditional and established algorithms between the nature inspired algorithms, inspired from the biological evolution in nature. In the passed year EA are extensively used for solving various specializations of science and real-time applications to find an optimum solution for complex and optimization problems [201] The word Evolutionary algorithm is used to define a collection of optimization techniques that simulate the natural biological evolution and the social behavior of living species. Differential Evolution (DE), Evolutionary strategy (ES), Genetic algorithm (GA), Genetic programming (GP), and Granular Agent Evolutionary Algorithm are the most well-known Algorithms belongs to Evolutionary algorithms (EAs) [201] [202]. 


\subsection{Swarm intelligence (SI)}

In computer sciences, Swarm Intelligence (SI) is the field of studying and designing efficient computational methods to solve problems using the behavior of real swarms such as birds, fish, and ants [1][2][201]. SI is a part of Artificial Intelligence introduced in the global optimization framework in 1989 by Jing Wang and Gerardo Beni as a collection of algorithms for controlling robotic swarm [1][2].

Swarm Intelligence issued a number of homogenous agents which interacts with each other either directly or indirectly, they communicate directly with each other by using audio or visual tools, as the honey bees communicate by waggle dance; indirect communication refers to as stigmergy [1][2][3][202]. Grasse first introduced the concepts stigmergy to the processes when an insect makes change in the. environment around it, and the other insects respond to that change and adapt themselves. to the new environment, such as in ant colonies when an ant deposit pheromone in its way to the food, leads other ants to follow that way Examples of swarm intelligence methods are Ant Colony Optimization (ACO), Artificial Bee Colony (ABC), Particle Swarm Optimization (PSO), Bacterial Foraging, Artificial Immune System, Stochastic diffusion search, Cat Swarm Optimization, Gravitational search algorithm, Bat algorithm, and Glowworm Swarm Optimization [1][2][3][201].

\subsection{Local search methods}

Local search metaheuristics refers to a group of methods which depend on a neighborhood, which are a set of candidate states, that are connected directly to the current state and can be reached by a single move for finding a best solution for computationally hard optimization problems. Its methods are iteratively improve the ordering of current solution states by performing simple modifications on the current solutions to obtain a new solution, the improvement continues until some stopping conditions has been satisfied or when there is no better solution in the given neighborhood [2].

The most famous and widely used Local search algorithms is k-opt which takes an initial tour and improves it by making flips in the tour to obtain a better tour, it is the basics of 2-opt, 3-opt, and Lin-Kernighan (LK)[2]. A more complicated local search method that could have other Local search method imbedded inside it, is called Great Deluge Algorithm (GDA), its procedure is finding a better solution from the neighborhood of the initial solution, and then it iteratively improving solution. Another local search method which is based on Hill-Climbing, includes some kind of intelligent in it, and is known as Tabu Search (TS), its procedure based on saving the previous moves in a list called "tabu list" which is used to avoid cycling. Many other local search algorithms are used in computer field to escape from local optimality which take unbounded time, one of the most known algorithms is Simulated annealing (SA) [2].

\section{Historical study}

In the history Several problem-solving technique tend to be metaheuristic; however heuristic as a scientific technique for optimization is a modern phenomenon. Metaheuristic algorithm or heuristic algorithm are developed in different historical periods.

Between 1940s and 1960s, heuristic methods widely used in different applications, but the main achievement was in 1963 with the introduction of evolutionary algorithms (EA) by I. Rechenberg and H. Schwefel [9].

Between 1960s and 1970s, saw the development of Genetic algorithms by J. Holland in 1975 [10].

Between 1980s and 1990s found one of the biggest step in the metaheuristics algorithms which was the simulated annealing (SA) in 1983 by S. Kirkpatrick, D. Gelatt Jr., and M. P. Vecchi [11]. Another significant step was the development of artificial immune systems in 1986 by Farmer, Packard and Perelson [12].

in the 1986, for the first time memory was used in metaheuristics by Fred Glover in Tabu search (TS) algorithm in which the previous search moves are stored in a Tabu-list, and upcoming moves must avoid revisiting previous moves [13].

Between 1990s and 2000s, was an exciting period for metaheuristic algorithms, as it saw the development of several important algorithms such as ant colony optimization (ACO) by Marco Dorigo in 1992 [7, 1, 4], and particle swarm optimization (PSO) by James Kennedy and Russell C. Eberhart in 1995 [1, 2, 3, 14], and later differential evolution (DE) was developed by Storn, R.; Price, K in 1997 [15].

Form 2000 until nowadays, metaheuristic algorithms has widely used in many applications, and many new significant algorithms has been developed. In 2001 Zong Woo Geem, Joong Hoon Kim, and G. V. developed the harmony search (HS) algorithm [16]. in 2002, a bacteria foraging algorithm was developed by K.M. Passino [17].

in 2005 D. Karaboga developed the artificial bee colony (ABC). In 2009, Xin-She Yang and Suash Deb developed cuckoo search (CS) algorithm [18].

Generally there are many significant metaheuristic algorithms that have been developed to solve real life and optimizations problems [3] [4]. Table 1: Shows Some of Metaheuristic Algorithms with Its Authors Name and Establishing Year.

Table 1: List of Metaheuristic Algorithms.

\begin{tabular}{|c|c|c|c|c|c|}
\hline ID & Year & Algorithm Name & Abbreviation & Authors & References \\
\hline 1. & 1961 & Pattern Search & PS & R. Hooke, T. A. Jeeves & [19] \\
\hline 2. & 1963 & evolutionary algorithms & EA & Rechenberg \& Schwefel & [9] \\
\hline 3. & 1965 & Evolution Strategy & ES & Rechenberg \& Schwefel & [9] \\
\hline 4. & 1966 & Evolutionary Programming & EP & David B. Fogel, Lawrence J. Fogel & [20] \\
\hline 5. & 1975 & Genetic Algorithm & GA & John Holland & [10] [203] \\
\hline 6. & 1977 & Scatter Search & SS & F. Glover & [21] \\
\hline 7. & 1983 & Simulated Annealing & SA & S. Kirkpatrick, D. Gelatt Jr., and M. P. Vecchi & [11] \\
\hline 8. & 1986 & Tabu Search & TS & Fred Glover & [13] [2] [203] \\
\hline 9. & 1986 & artificial immune systems & AIS & Farmer, Packard and Perelson & [12] \\
\hline 10. & 1989 & Stochastic Diffusion Search & SDS & Bishop, J.M. & [22] \\
\hline 11. & 1989 & Memetic Algorithm & MA & Moscato & [23] \\
\hline 12. & 1992 & Ant Colony Optimization & $\mathrm{ACO}$ & A. Colorni, M. Dorigo, V. Maniezzo & [2] [24] \\
\hline 13. & 1993 & Great Deluge Algorithm & GDA & G. Dueck & [2] [25] [195] \\
\hline 14. & 1995 & Particle Swarm Optimization & PSO & R. Eberhart, J. Kennedy & [3] [14][203] \\
\hline 15. & 1997 & Differential Evolution & $\mathrm{DE}$ & Storn, R.; Price, K & [15] \\
\hline 16. & 1998 & $\begin{array}{l}\text { Photosynthetic Learning Algo- } \\
\text { rithm }\end{array}$ & PLA & Haruhiko Murase, Akira Wadano & [26] \\
\hline
\end{tabular}




\begin{tabular}{|c|c|c|c|c|c|}
\hline 17. & 2000 & Clonal Selection Algorithm & CSA & L.N. de Castro, F.J. von Zuben & [27] \\
\hline 18. & 2001 & Harmony Search & HS & Zong Woo Geem, Joong Hoon Kim, and G.V. & [16] \\
\hline 19. & 2001 & $\begin{array}{l}\text { Honey-bees Mating Optimization } \\
\text { Algorithm }\end{array}$ & HBMOA & H.A. Abbass & {$[28]$} \\
\hline 20. & 2001 & $\begin{array}{l}\text { POPMUSIC: Partial Optimization } \\
\text { Metaheuristic Under Special } \\
\text { Intensification }\end{array}$ & POPMUSIC & Éric D. Taillard, Stefan Voss & [29] \\
\hline 21. & 2001 & Sheep Flocks Heredity Model & SFHM & Hyunchul Kim and Byungchul Ahn & {$[30]$} \\
\hline 22. & 2002 & Artificial Fish School Algorithm & AFS & X.L. Li, Z.J. Shao and J.X. Qian & [31] \\
\hline 23. & 2002 & Bacteria Chemotaxis Algorithm & BCA & $\begin{array}{l}\text { S.D. Muller, J. Marchetto, S. Airaghi and P. Kournoutsakos, } \\
2002\end{array}$ & {$[32]$} \\
\hline 24. & 2002 & Bacterial Foraging Algorithm & BFA & K.M. Passino & [17] \\
\hline 25. & 2002 & Gene Expression Programming & & C. Ferreira & [33] \\
\hline 26. & 2002 & Social Cognitive Optimization & $\mathrm{SCO}$ & Xiao-Feng Xie, Wen-Jun Zhang, Zhi-Lian Yang & [34] \\
\hline 27. & 2002 & Bacterial Foraging Optimization & $\mathrm{BFO}$ & Passino & [35] \\
\hline 28. & 2003 & Shuffled Frog Leaping Algorithm & SFL & Eusuff and K.E. Lansey & [36] \\
\hline 29. & 2003 & Gravitational Search Algorithm & GSA & B. Webster and P.J. Bernhard & [37] \\
\hline 30. & 2003 & Queen-bee Evolution & GBE & S.H. Jung & {$[38]$} \\
\hline 31. & 2003 & Society and Civilization & $\mathrm{SC}$ & Ray, Tapabrata, and Kim Meow Liew & [39] \\
\hline 32. & 2003 & Fish Swarm Algorithm & FSA & Li, Shao, Qian & [40] \\
\hline 33. & 2005 & Wasp Swarm Optimization & WSO & P Pinto, TA Runkler, JM Sousa & [41] \\
\hline 34. & 2006 & Big bang-big Crunch & $\mathrm{BBBC}$ & Osman K. Erol and Ibrahim Eksin & [42] \\
\hline 35. & 2006 & Cat Swarm Optimization & $\mathrm{CSO}$ & Shu-Chuan Chu, Pei-Wei Tsai, and Jeng-Shyang Pan & [43] \\
\hline 36. & 2006 & $\begin{array}{l}\text { Covariance Matrix Adaptation- } \\
\text { Evolution Strategy }\end{array}$ & CMAES & Nikolaus Hansen, Sibylle D. Müller and Petros Koumoutsakos & [44] \\
\hline 37. & 2006 & Invasive Weed Optimization & IWO & A.R. Mehrabian, C. Lucas & [45] \\
\hline 38. & 2006 & Saplings Growing Up Algorithm & SGUO & Ali Karci, Bilal Alatas & [46] \\
\hline 39. & 2006 & $\begin{array}{l}\text { Small-world Optimization Algo- } \\
\text { rithm }\end{array}$ & SWOA & H Du, X Wu, J Zhuang & [47] \\
\hline 40. & 2006 & $\begin{array}{l}\text { Invasive Weed Colony Optimiza- } \\
\text { tion }\end{array}$ & IWCO & Mehrabian, Lucas & [48] \\
\hline 41. & 2006 & Group Search Optimization & GSO & $\mathrm{He}, \mathrm{Wu}$, Saunders & [49] \\
\hline 42. & 2007 & Artificial Bee Colony Algorithm & $\mathrm{ABC}$ & Dervis Karaboga and Bahriye & [3] [50] \\
\hline 43. & 2007 & Central Force Optimization & $\mathrm{CFO}$ & Richard A. Formato & [51] \\
\hline 44. & 2007 & Good Lattice Swarm Algorithm & GLSA & Shoubao Su, Jiwen Wang, Wangkang Fan, Xibing Yin & [52] \\
\hline 45. & 2007 & $\begin{array}{l}\text { Imperialist Competitive Algo- } \\
\text { rithm }\end{array}$ & ICA & Esmaeil Atashpaz-Gargari and Caro & [53] \\
\hline 46. & 2007 & Monkey Search & MS & Antonio Mucherino and Onur Seref & [54] \\
\hline 47. & 2007 & $\begin{array}{l}\text { Parliamentary Optimization Al- } \\
\text { gorithm }\end{array}$ & POA & A. Borji & {$[55]$} \\
\hline 48. & 2007 & River Formation Dynamics & RFD & P. Rabanal, I. Rodríguez, F. Rubio & {$[56]$} \\
\hline 49. & 2007 & Simplex Heuristic & $\mathrm{SH}$ & J.P. Pedroso & [57] \\
\hline 50. & 2008 & Bacterial Swarming & BS & Ying Chu, Hua Mi and Huilian Liao & [58] \\
\hline 51. & 2008 & $\begin{array}{l}\text { Biogeography Based Optimiza- } \\
\text { tion }\end{array}$ & $\mathrm{BBO}$ & Ying Chu, Hua Mi and Huilian Liao & [59] \\
\hline 52. & 2008 & Fish-school Search & FS & $\begin{array}{l}\text { Carmelo J. A. Bastos Filho, Fernando B. de Lima Neto, Anthony } \\
\text { J. C. C. Lins, Antonio I. S. Nascimento, Marilia P. Lima }\end{array}$ & {$[60]$} \\
\hline 53. & 2008 & Plant Growth Optimisation & PGO & Wei Cai, Weiwei Yang and Xiaoqian Chen & {$[61]$} \\
\hline 54. & 2008 & Roach Infestation Optimization & RIO & $\begin{array}{l}\text { Timothy C. Havens, Christopher J. Spain, Nathan G. Salmon and } \\
\text { James M. Keller }\end{array}$ & {$[62]$} \\
\hline 55. & 2008 & Viral Systems & VS & Cortés P., García J.M., Muñuzuri J. and Onieva L0 & {$[63]$} \\
\hline 56. & 2009 & $\begin{array}{l}\text { Artificial Searching Swarm Algo- } \\
\text { rithm }\end{array}$ & ASSA & T. Chen & [64] \\
\hline 57. & 2009 & Bacterial Evolutionary Algorithm & BEA & Swagatam Das, Archana Chowdhury and Ajith Abraham & {$[65]$} \\
\hline 58. & 2009 & Consultant-guided Search & CGS & S. Iordache & [66] \\
\hline 59. & 2009 & Dialectic Search & DS & S Kadioglu, Meinolf Sellmann & [67] \\
\hline 60. & 2009 & Dolphin Partner Optimization & DPO & Y Shiqin, J Jianjun, Y Guangxing & [68] \\
\hline 61. & 2009 & Firefly Algorithm & FA & Xin-She Yang & [69] \\
\hline 62. & 2009 & Glowworm Swarm Optimization & GSO & K. N. Krishnanand and Debasish Ghose & [70] \\
\hline 63. & 2009 & Group Search Optimizer & GSO & S. He, Q.H. Wu, J.R. Saunders & [71] \\
\hline 64. & 2009 & Human-inspired Algorithm & HIA & L.M. Zhang, C. Dahlmann, Y. Zhang & [72] \\
\hline 65. & 2009 & Hunting Search & HS & R. Oftadeh, M. J. Mahjoob & [73] \\
\hline 66. & 2009 & $\begin{array}{l}\text { Intelligent Water Drops Algo- } \\
\text { rithm }\end{array}$ & IWDA & Hamed Shah-Hosseini & [74] \\
\hline 67. & 2009 & League Championship Algorithm & LCA & Ali Husseinzadeh Kashan & [75] \\
\hline 68. & 2009 & Locust Swarms & LS & Stephen Chen & [76] \\
\hline 69. & 2009 & Paddy Field Algorithm & PFA & U. Premaratne, J. Samarabandu, T. Sidhu & [77] \\
\hline 70. & 2009 & $\begin{array}{l}\text { Bumble Bees Mating Optimiza- } \\
\text { tion }\end{array}$ & BBMO & Marinakis, Marinaki, Matsatsinis & {$[78]$} \\
\hline 71. & 2009 & Hunting Search Optimization & HSO & Oftadeh, Mahjoob & [79] \\
\hline 72. & 2009 & Cuckoo Search & CS & Yang, Deb & [18] \\
\hline 73. & 2010 & Bat Algorithm & BA & Xin-She Yang & [3] [80] \\
\hline 74. & 2010 & Charged System Search & CSS & A. Kaveh, and S. Talatahari & [81] \\
\hline 75. & 2010 & Eagle Strategy & ES & X.S. Yang, S. Deb & {$[82]$} \\
\hline 76. & 2010 & Fireworks Algorithm & FA & Y. Tan, Y. Zhu & [83] \\
\hline 77. & 2010 & Grenade Explosion Method & GEM & Ali Ahrari and Ali A. Atai & [84] \\
\hline 78. & 2010 & Group Counseling Optimization & GCO & M.A. Eita and M. M. Fahmy & {$[85]$} \\
\hline 79. & 2010 & Hierarchical Swarm Model & HSM & Hanning Chen, Yunlong Zhu, Kunyuan $\mathrm{Hu}$ and Xiaoxian He & [86] \\
\hline 80. & 2010 & Reincarnation Algorithm & RA & A. Sharma & [87] \\
\hline 81. & 2010 & Social Emotional Optimization & SEO & Y. Xu, Z. Cui, J. Zeng & [88] \\
\hline
\end{tabular}




\begin{tabular}{|c|c|c|c|c|c|}
\hline 82. & 2010 & Termite Colony Optimization & $\mathrm{TCO}$ & $\begin{array}{l}\text { Ramin Hedayatzadeh, Foad Akhavan Salmassi, Reza Akbari, } \\
\text { Koorush }\end{array}$ & [89] \\
\hline 83. & 2010 & Wind Driven Optimization & WDO & Zikri Bayraktar, Muge Komurcu, and Douglas H. Werner & {$[90]$} \\
\hline 84. & 2010 & Bat Inspired Approach & BIA & Yang & [91] \\
\hline 85. & 2011 & $\begin{array}{l}\text { Artificial Chemical Reaction } \\
\text { Optimization Algorithm }\end{array}$ & ACROA & Bilal Alatas & {$[92]$} \\
\hline 86. & 2011 & $\begin{array}{l}\text { Eco-inspired Evolutionary Algo- } \\
\text { rithm }\end{array}$ & EIEA & R.S. Parpinelli, H.S. Lopes & {$[93]$} \\
\hline 87. & 2011 & Galaxy-based Search Algorithm & GBS & Hamed Shah-Hosseini & [94] \\
\hline 88. & 2011 & Migrating Birds Optimization & $\mathrm{MBO}$ & Ekrem Duman, Mitat Uysal, Ali Fuat Alkaya & [95] \\
\hline 89. & 2011 & Plant Propagation Algorithm & PPA & Abdellah Salhi, Eric S Fraga & [96] \\
\hline 90. & 2011 & $\begin{array}{l}\text { Spiral Dynamics Inspired Opti- } \\
\text { mization }\end{array}$ & SDIO & K. Tamura, K. Yasuda & [97] \\
\hline 91. & 2011 & $\begin{array}{l}\text { Teaching-learning based Optimi- } \\
\text { zation }\end{array}$ & TLBO & R. V. Rao, V. J. Savsani, D. P. Vakharia & {$[98]$} \\
\hline 92. & 2011 & Water-flow Algorithm & WFA & Trung Hieu Tran, Kien Ming Ng & [99] \\
\hline 93. & 2012 & Anarchic Society Optimization & ASO & H. Shayeghi and J. Dadashpour & [100] \\
\hline 94. & 2012 & $\begin{array}{l}\text { Artificial Plant Optimization } \\
\text { Algorithm }\end{array}$ & APO & Jun Li, Zhihua Cui and Zhongzhi Shi & [101] \\
\hline 95. & 2012 & Bacterial Colony Optimization & $\mathrm{BCO}$ & Ben Niu and Hong Wang & [102] \\
\hline 96. & 2012 & $\begin{array}{l}\text { Community of Scientist Optimi- } \\
\text { zation }\end{array}$ & $\mathrm{CSO}$ & Alfredo Milani and Valentino Santucci & {$[103]$} \\
\hline 97. & 2012 & Differential Search Algorithm & $\mathrm{DF}$ & P. Civicioglu & [104] \\
\hline 98. & 2012 & Electro-magnetism Optimization & EM & $\begin{array}{l}\text { Erik Cuevas, Diego Oliva, Daniel Zaldivar, Marco Pérez- } \\
\text { Cisneros, Humberto }\end{array}$ & {$[105]$} \\
\hline 99. & 2012 & Flower Pollination Algorithm & FPA & Xin-She Yang & [106] \\
\hline 100. & 2012 & Fruit Fly Optimization Algorithm & FFO & W. T. Pan & [107] \\
\hline 101. & 2012 & Great Salmon Run & GSR & A. Mozaffari, A. Fathi, S. Behzadipour & [108] \\
\hline 102. & 2012 & Hoopoe Heuristic Optimization & $\mathrm{HHO}$ & $\begin{array}{l}\text { Mohammed El-Dosuky, Ahmed El-Bassiouny, Taher Hamza, and } \\
\text { Magdy Rashad }\end{array}$ & [109] \\
\hline 103. & 2012 & Japanese Tree Frogs Calling & JTFC & H. Hernández, C. Blum & [110] \\
\hline 104. & 2012 & Krill Herd & $\mathrm{KH}$ & Amir Hossein Gandomi and Amir Hossein Alavi & [111] \\
\hline 105. & 2012 & Mine Blast Algorithm & MBA & $\begin{array}{l}\text { Ali Sadollah, Ardeshir Bahreininejad, Hadi Eskandar, Mohd } \\
\text { Hamdi ( }\end{array}$ & {$[112]$} \\
\hline 106. & 2012 & Ray Optimization & RO & A. Kaveh and M. Khayatazad & [113] \\
\hline 107. & 2012 & Soccer Game Optimization & SGO & H.D. Purnomo, H.-M. Wee & [114] \\
\hline 108. & 2012 & Water Cycle Algorithm & WCA & $\begin{array}{l}\text { Hadi Eskandar, Ali Sadollah, Ardeshir Bahreininejad, Mohd } \\
\text { Hamdi, }\end{array}$ & {$[115]$} \\
\hline 109. & 2012 & Wolf Search Algorithm & WF & Rui Tang, S. Fong, Xin-She Yang, and S. Deb & [116] \\
\hline 110. & 2012 & Zombie Survival Optimization & $\mathrm{ZSO}$ & Hoang Thanh Nguyen, Bir Bhanu & [117] \\
\hline 111. & 2012 & Collective Animal Behavior & CBA & Cuevas,_González, Zaldivar, Pérez-Cisneros,_García__ & [118] \\
\hline 112. & 2013 & Dolphin Echolocation & $\mathrm{DE}$ & A. Kaveh, N. Farhoudi & [119] \\
\hline 113. & 2013 & African Wild Dog Algorithm & AWD & C. Subramanian, A.S.S. Sekar and K. Subramanian & [120] \\
\hline 114. & 2013 & Atmosphere Clouds Model & $\mathrm{ACM}$ & Gao Wei Yan and Zhan Ju Hao & {$[121]$} \\
\hline 115. & 2013 & $\begin{array}{l}\text { Backtracking Search Optimiza- } \\
\text { tion }\end{array}$ & BSO & Pinar Civicioglu & {$[122]$} \\
\hline 116. & 2013 & Black Holes Algorithm & BHA & Abdolreza Hatamlou & [123] \\
\hline 117. & 2013 & $\begin{array}{l}\text { Blind, Naked Mole-rats Algo- } \\
\text { rithm }\end{array}$ & BNMR & $\begin{array}{l}\text { Mohammad Taherdangkoo, Mohammad Hossein Shirzadi, } \\
\text { Mehran Yazdi } \\
\text { and Mohammad Hadi Bagheri }\end{array}$ & [124] \\
\hline 118. & 2013 & Cuttlefish Algorithm & CFA & $\begin{array}{l}\text { Adel Sabry Eesa, Adnan Mohsin Abdulazeez Brifcani and } \\
\text { Zeynep Orman }\end{array}$ & [125] \\
\hline 119. & 2013 & Egyptian Vulture Optimization & EVO & Chiranjib Sur, Sanjeev Sharma, and Anupam Shukla & {$[126]$} \\
\hline 120. & 2013 & $\begin{array}{l}\text { Gases Brownian Motion Optimi- } \\
\text { zation }\end{array}$ & GBMO & M. Abdechiri, M.R. Meybodi, H. Bahrami & [127] \\
\hline 121. & 2013 & $\begin{array}{l}\text { Magnetotactic Bacteria Optimiza- } \\
\text { tion Algorithm }\end{array}$ & MBOA & Hongwei Mo and Lifang Xu & [128] \\
\hline 122. & 2013 & $\begin{array}{l}\text { Penguins Search Optimization } \\
\text { Algorithm }\end{array}$ & PSOA & Y. Gheraibia, A. Moussaoui & [129] \\
\hline 123. & 2013 & $\begin{array}{l}\text { Seven-spot Ladybird Optimiza- } \\
\text { tion }\end{array}$ & SLO & Peng Wang, Zhouquan Zhu, and Shuai Huang & {$[14]$} \\
\hline 124. & 2013 & Social Spider Algorithm & SSA & $\begin{array}{l}\text { Erik Cuevas, Miguel Cienfuegos, Daniel Zaldívar, Marco Pérez- } \\
\text { Cisneros }\end{array}$ & [130] \\
\hline 125. & 2013 & $\begin{array}{l}\text { Swallow Swarm Optimization } \\
\text { Algorithm }\end{array}$ & SSO & Mehdi Neshat, Ghodrat Sepidnam & [131] \\
\hline 126. & 2014 & $\begin{array}{l}\text { Animal Migration Optimization } \\
\text { Algorithm }\end{array}$ & AMO & Xiangtao Li, Jie Zhang and Minghao Yin & [132] \\
\hline 127. & 2014 & Artificial Ecosystem Algorithm & AEA & Manal T. Adham and Peter J. Bentley & [133] \\
\hline 128. & 2014 & Bird Mating Optimizer & BMO & Alireza Askarzadeh & [134] \\
\hline 129. & 2014 & Chicken Swarm & CS & Xianbing Meng, Yu Liu, Xiaozhi Gao, Hengzhen Zhang & [135] \\
\hline 130. & 2014 & Cockroach Swarm Optimization & $\mathrm{CSO}$ & I. C. Obagbuwa and A. O. Adewumi & [136] \\
\hline 131. & 2014 & Colliding Bodies Optimization & $\mathrm{CBO}$ & A. Kaveh and V. R. Mahdavi & [137] \\
\hline 132. & 2014 & $\begin{array}{l}\text { Coral Reefs Optimization Algo- } \\
\text { rithm }\end{array}$ & $\mathrm{CRO}$ & $\begin{array}{l}\text { S. Salcedo-Sanz, J. Del Ser, I. Landa-Torres, S. Gil-López, and J. } \\
\text { A. Portilla-Figueras }\end{array}$ & {$[138]$} \\
\hline 133. & 2014 & Exchange Market Algorithm & EMA & N. Ghorbani, E. Babaei & [139] \\
\hline 134. & 2014 & Forest Optimization Algorithm & FOA & Manizheh Ghaemi, Mohammad-Reza Feizi-Derakhshi & [140] \\
\hline 135. & 2014 & Golden Ball & GB & E. Osaba, F. Diaz, E. Onieva & [141] \\
\hline 136. & 2014 & Greedy Politics Optimization & GPO & J.M.L. Melvix & [142] \\
\hline 137. & 2014 & Grey Wolf Optimizer & GWO & $\begin{array}{l}\text { Seyedali Mirjalili, Seyed Mohammad Mirjalili, and Andrew Lew- } \\
\text { is }\end{array}$ & {$[143]$} \\
\hline
\end{tabular}




\begin{tabular}{|c|c|c|c|c|}
\hline 138. 2014 & Heart & $\mathrm{H}$ & Abdolreza Hatamlou & {$[144]$} \\
\hline 139. 2014 & Interior Design and Decoration & IDD & A.H. Gandomi & [145] \\
\hline 140. 2014 & Kaizen Programming & $\mathrm{KP}$ & V. V. Melo & [146] \\
\hline 141. 2014 & Keshtel Algorithm & KA & M. Hajiaghaei-Keshteli, M. Aminnayeri & {$[147]$} \\
\hline 142. 2014 & $\begin{array}{l}\text { Raven Roosting Optimization } \\
\text { Algorithm }\end{array}$ & RROA & Anthony Brabazon, Wei Cui, Michael O’Neill & [148] \\
\hline 143. 2014 & $\begin{array}{l}\text { Scientific Algorithms for the Car } \\
\text { Renter Salesman Problem }\end{array}$ & & D. Felipe, E. Goldbarg, and M. Goldbarg & [149] \\
\hline 144. 2014 & Shark Smell Optimization & SSO & Oveis Abedinia, Nima Amjady, and Ali Ghasemi & {$[150]$} \\
\hline 146. 2014 & Strawberry Algorithm & SA & F. Merrikh-Bayat & [152] \\
\hline 147. 2014 & Symbiotic Organisms Search & SOS & M.Y. Cheng, D. Prayogo & {$[153]$} \\
\hline 148. 2014 & Worm Optimization & WO & J.P. Arnaout & [154] \\
\hline 149. 2015 & Ant Lion & $\mathrm{AL}$ & S. Mirjalili & {$[155]$} \\
\hline 150. 2015 & Artificial Algae Algorithm & AAA & Sait Ali, Uymaz, GulayTezel and Esra Yel & {$[156]$} \\
\hline 151. 2015 & $\begin{array}{l}\text { U-Turning Ant Colony Optimiza- } \\
\text { tion }\end{array}$ & U-TACO & Saman M. Almufti & {$[2][4][202][203]$} \\
\hline 153. 2015 & Bull Optimization Algorithm & BOA & $\begin{array}{l}\text { Sait Ali, Uymaz, GulayTezel and } \\
\text { Esra Yel }\end{array}$ & {$[158]$} \\
\hline 154. 2015 & Elephant Herding Optimization & $\mathrm{EHO}$ & Gai-Ge Wang, Suash Deb, Leandro dos S. Coelho & {$[1][159][202]$} \\
\hline 155. 2015 & Elephant Search Algorithm & ESA & Suash Deb, Simon Fong, and Zhonghuan Tian & {$[160]$} \\
\hline 156. 2015 & $\begin{array}{l}\text { General Relativity Search Algo- } \\
\text { rithm }\end{array}$ & GRSA & Hamzeh Beiranvand, Esmaeel Rokrok & {$[161]$} \\
\hline 157. 2015 & $\begin{array}{l}\text { Invasive Tumor Growth Optimi- } \\
\text { zation Algorithm }\end{array}$ & ITGOA & D Tang, S Dong, Y Jiang, H Li, Y Huang & {$[162]$} \\
\hline 158. 2015 & Ions Motion Algorithm & IMA & B. Javidy, A. Hatamlou, S. Mirjalili & {$[163]$} \\
\hline 159. 2015 & $\begin{array}{l}\text { Jaguar Algorithm with Learning } \\
\text { Behavior }\end{array}$ & JA & $\begin{array}{l}\text { Chin-Chi Chen, Yung-Che Tsai, I-I Liu, Chia-Chun Lai, Yi-Ting } \\
\text { Yeh, Shu-Yu Kuo, Yao-Hsin Chou }\end{array}$ & [164] \\
\hline 160. 2015 & Lightning Search Algorithm & LSA & H. Shareef, A.A. Ibrahim, A.H. Mutlag & {$[165]$} \\
\hline 161. 2015 & Monarch Butterfly Optimization & $\mathrm{MBO}$ & G. Wang, S. Deb, Z. Cui & {$[166]$} \\
\hline 162. 2015 & $\begin{array}{l}\text { Moth-flame Optimization Algo- } \\
\text { rithm }\end{array}$ & MFOA & Seyedali Mirjalili & {$[167]$} \\
\hline 163. 2015 & Multi-verse Optimizer & MVO & S. Mirjalili, S. M. Mirjalili, A. Hatamlou & [168] \\
\hline 164. 2015 & Optics Inspired Optimization & $\mathrm{OIO}$ & A.H. Kashan & [169] \\
\hline 167. 2015 & Stochastic Fractal Search & SFS & H. Salimi & [172] \\
\hline 168. 2015 & Vortex Search Algorithm & VSA & B. Dogan, T. Olmez & {$[173]$} \\
\hline 169. 2015 & Water Wave Optimisation & WWO & Y.J. Zheng & {$[174]$} \\
\hline 170. 2016 & African Buffalo Optimization & $\mathrm{ABO}$ & J.B. Odili and M.N. Mohmad Kahar & {$[175]$} \\
\hline 171. 2016 & Bird Swarm & BS & $\begin{array}{l}\text { Xian-Bing Meng, X.Z. Gao, Lihua Lu, Yu Liu \& Hengzhen } \\
\text { Zhang }\end{array}$ & {$[176]$} \\
\hline 172. 2016 & Camel Algorithm & CA & M. K. Ibrahim, R. S. Ali & {$[177]$} \\
\hline 173. 2016 & $\begin{array}{l}\text { Crystal Energy Optimization } \\
\text { Algorithm }\end{array}$ & CEOA & X. Feng, M. Ma, and H. Yu & {$[178]$} \\
\hline 174. 2016 & Dragonfly Algorithm & DA & S. Mirjalili & {$[179]$} \\
\hline 175. 2016 & FIFA World Cup & FWC & N. Razmjooy, M. Khalilpour, M. Ramezani & {$[180]$} \\
\hline 176. 2016 & Flying Elephants Algorithm & FEA & Adilson Elias Xavier, Vinicius Layter Xavier & {$[181]$} \\
\hline 177. 2016 & Lion Optimization Algorithm & LOA & Maziar Yazdani and Fariborz Jolai & {$[182]$} \\
\hline 178. 2016 & $\begin{array}{l}\text { Rooted Tree Optimization Algo- } \\
\text { rithm }\end{array}$ & RTOA & $\begin{array}{l}\text { Yacine Labbi, Djilani Ben Attous, Hossam A. Gabbar, Belkacem } \\
\text { Mahdad, Aboelsood }\end{array}$ & {$[183]$} \\
\hline 179. 2016 & Sperm Whale Algorithm & SWA & A. Ebrahimi, E. Khamehchi & {$[184]$} \\
\hline 180. 2016 & Virus Colony Search & VCS & Mu Dong Li, Hui Zhao, Xing Wei Weng, Tong Han & {$[185]$} \\
\hline 181. 2016 & Virus Optimization Algorithm & VOA & Chia Liang, Josue Rodolfo Cuevas & [186] \\
\hline 182. 2016 & Water Evaporation Optimization & WEO & A. Kaveh and T. Bakhshpoori & {$[187]$} \\
\hline 183. 2016 & Whale Optimization Algorithm & WOA & Seyedali Mirjalili and Andrew Lewisa & {$[188]$} \\
\hline 184. 2017 & $\begin{array}{l}\text { Grasshopper Optimisation Algo- } \\
\text { rithm }\end{array}$ & GOA & Shahrzad Saremi, Seyedali Mirjalili, Andrew Lewis & [189] \\
\hline 185. 2017 & Sperm Motility Algorithm & SMA & Raouf, Hezam & {$[190]$} \\
\hline 186. 2017 & Rain Water Algorithm & RWA & $\begin{array}{l}\text { Biyanto, T R; Matradji; Syamsi, M N; Fibrianto, H Y; Afdanny, } \\
\text { N; Rahman, A H; Gunawan, K S; Pratama, J A D; Malwindasari, } \\
\text { A; Abdillah, A I; Bethiana, T N; Putra, Y A }\end{array}$ & [195] \\
\hline 187. 2017 & Hydrological Cycle Algorithm & $\mathrm{HCA}$ & Wedyan, Ahmad; Whalley, Jacqueline; Narayanan, Ajit & [197] \\
\hline 188. 2018 & Farmland fertility & FF & H Shayanfar, F Gharehchopogh & [191] \\
\hline 189. 2019 & The Sailfish Optimize & TSO & S Shadravan, H Naji, V Bardsiri, & [192] \\
\hline 190. 2019 & $\begin{array}{l}\text { Heterogeneous pigeon-inspired } \\
\text { optimization }\end{array}$ & HPIO & Hao WangZhuxi ZhangZhen DaiJun ChenXi Zhu & {$[193]$} \\
\hline 191. 2019 & Harris hawks optimization & $\mathrm{HHO}$ & $\begin{array}{l}\text { Heidari, Ali Asghar; Mirjalili, Seyedali; Faris, Hossam; Aljarah, } \\
\text { Ibrahim; Mafarja, Majdi; Chen, Huiling }\end{array}$ & [196] \\
\hline 192. 2019 & Emperor Penguins Colony & EPC & $\begin{array}{l}\text { Harifi, Sasan; Khalilian, Madjid; Mohammadzadeh, Javad; } \\
\text { Ebrahimnejad, Sadoullah }\end{array}$ & [198] \\
\hline
\end{tabular}

\section{Summary}

In the real life the daily problems are becoming more and more complex in a way that it become very difficult for a traditional methods to solve them within a reasonable time. 
Metaheuristics algorithm have been used to solve the real-life problems in an optimal time and effort. In the past many algorithm have been developed that belongs to metaheuristic algorithms.

This paper is an attempt to provide a historical list of some of metaheuristic algorithms that have been used between 1961 and 2019 , it provides the year of establishments, authors name, abbreviations and the reference of the algorithm.

\section{References}

[1] S. Almufti, R. Asaad and B. Salim, "Review on Elephant Herding Optimization Algorithm Performance in Solving Optimization Problems", Sciencepubco.com, 2019. [Online]. Available: https://www.sciencepubco.com/index.php/ijet/article/view/28473. [Accessed: 26- May- 2019].

[2] S. Almufti, "U-Turning Ant Colony Algorithm powered by Great Deluge Algorithm for the solution of TSP Problem", Hdl.handle.net, 2018. [Online].

[3] S. Almufti, "Using Swarm Intelligence for solving NPHard Problems," Academic Journal of Nawroz University, vol. 6, no. 3, pp. 46-50, 2017. https://doi.org/10.25007/ajnu.v6n3a78.

[4] S. Almufti and A. Shaban, "U-Turning Ant Colony Algorithm for Solving Symmetric Traveling Salesman Problem", Academic Journal of Nawroz University, vol. 7, no. 4, pp. 45-49, 2018. https://doi.org/10.25007/ajnu.v6n4a270.

[5] F. Glover, "Future paths for integer programming and links to artificial intelligence", Computers \& Operations Research, vol. 13, no. 5, pp. 533549, 1986. Available: 10.1016/0305-0548(86)90048-1. https://doi.org/10.1016/0305-0548(86)90048-1.

[6] F. Glover and M. Laguna, Tabu search. Boston, Mass.: Kluwer academic, 1998. https://doi.org/10.1007/978-1-4615-6089-0.

[7] C. Blum and A. Roli, "Metaheuristics in combinatorial optimization", ACM Computing Surveys, vol. 35, no. 3, pp. 268-308, 2003. Available: 10.1145/937503.937505. https://doi.org/10.1145/937503.937505.

[8] G. Dhiman and V. Kumar, "Spotted hyena optimizer: A novel bio-inspired based metaheuristic technique for engineering applications", Advances in Engineering Software, vol. 114, pp. 48-70, 2017. Available: 10.1016/j.advengsoft.2017.05.014. https://doi.org/10.1016/j.advengsoft.2017.05.014.

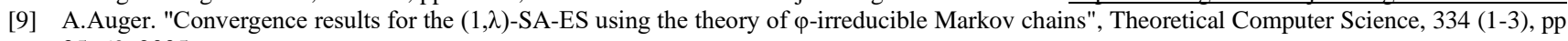
$35-69,2005$

[10] D. E. Goldberg. "Genetic Algorithms in Search, Optimization, and Machine Learning”, ADDISON-WESLEY PUBLISHING COMPANY, 1989

[11] S. Kirkpatrick, D. Gelatt Jr., and M. P. Vecchi, "Optimization by simulated annealing", Science, 220(4598), pp 671-680, 1983 https://doi.org/10.1126/science.220.4598.671.

[12] Farmer, N. Packard and A. Perelson, "The immune system, adaptation, and machine learning", Physica D: Nonlinear Phenomena, vol. 22, no. 1-3, pp. 187-204, 1986. Available: 10.1016/0167-2789(86)90240-x. https://doi.org/10.1016/0167-2789(86)90240-X.

[13] F. Glover. "Future Paths for Integer Programming and Links to Artificial Intelligence", Computers and Operations Research, 13 (5), pp 533-549, 1986. https://doi.org/10.1016/0305-0548(86)90048-1.

[14] R. Eberhart, J. Kennedy. “A New Optimizer Using Particle Swarm Theory”, In proceedings of the Sixth International Symposium on Machine and Human Science, pp. 39-43, 1995

[15] R. Storn, K. Price. "Differential evolution - a simple and efficient heuristic for global optimization over continuous spaces", Journal of Global Optimization, 11(4), pp 341-359, 1997 https://doi.org/10.1023/A:1008202821328.

[16] Z. W. Geem, J. H. Kim, G. V. Loganathan. "A new heuristic optimization algorithm: harmony search", Simulation, 76(2), pp 60-68, 2001 https://doi.org/10.1177/003754970107600201.

[17] K.M. Passino. "Biomimicry of bacterial foraging for distributed optimization and control", IEEE control systems, 22(3), pp 52-67, 2002 https://doi.org/10.1109/MCS.2002.1004010.

[18] X. S. Yang, S. Deb. "Cuckoo Search via Lévy flights”, In proceedings of 2009 World Congress on Nature \& Biologically Inspired Computing, Coimbatore, India, pp 210-214, 2009 https://doi.org/10.1109/NABIC.2009.5393690.

[19] R. Hooke, T. A. Jeeves. "Direct search" solution of numerical and statistical problems", Journal of the Association for Computing Machinery (ACM). 8 (2), pp 212-229, 1961 https://doi.org/10.1145/321062.321069.

[20] D. B. Fogel, L. J. Fogel. “An introduction to evolutionary programming”, In Proceedings of European Conference on Artificial Evolution, pp 21-33, 1995 https://doi.org/10.1007/3-540-61108-8_28.

[21] F. Glover. "Heuristics for Integer Programming Using Surrogate Constraints", Decision Sciences, 8, pp 156-166, 1977 https://doi.org/10.1111/j.1540-5915.1977.tb01074.x.

[22] Bishop, J.M., "Stochastic Searching Networks", Proc. 1st IEE Conf. on Artificial Neural Networks, London, pp 329-331, 1989

[23] P. Moscato, On Evolution, Search, Optimization, Genetic Algorithms and Martial Arts - Towards Memetic Algorithms. 1989.

[24] A.Colorni, M. Dorigo, V. Maniezzo. "Distributed Optimization by Ant Colonies", In the proceedings of the First European Conference on Artificial Life, Paris, France, Elsevier Publishing, 134-142, , 1991

[25] G. Dueck. "New Optimization Heuristics The Great Deluge Algorithm and the Record-to-Record Travel", Journal of Computational Physics, 104(1), pp 86-92, 1993 https://doi.org/10.1006/jcph.1993.1010.

[26] H. Murase, A. Wadano. "Photosynthetic Algorithm for Machine Learning and TSP", IFAC Proceedings Volumes, 31(12), pp 19-24, 1998 https://doi.org/10.1016/S1474-6670(17)36035-4.

[27] L.N. de Castro, F.J. von Zuben. "The clonal selection algorithm with engineering applications", In Proceedings of the Genetic and Evolutionary Computation Conference, Las Vegas, Nevada, USA, pp 36-39, 2000

[28] H.A. Abbass. "MBO: Marriage in honey bees optimization - A haplometrosis polygynous swarming approach" In proceedings of the IEEE Congress on Evolutionary Computation, Vol. 1, pp 207-214, 2001

[29] É. D. Taillard, S. Voss. "Popmusic - Partial Optimization Metaheuristic under Special Intensification Conditions", Essays and Surveys in Metaheuristics, Operations Research/Computer Science Interfaces Series, 15, pp 613-629, 2001 https://doi.org/10.1007/978-1-4615-1507-4 27.

[30] H. Kim, B. Ahn. "A new evolutionary algorithm based on sheep flocks heredity model", In Proceedings of the IEEE Pacific Rim Conference on Communications, Computers and signal Processing, PACRIM, vol. 2, pp 514-517, 2001

[31] X. L. Li, Z. J. Shao, J. X. Qian. “An optimizing method based on autonomous animals: Fish-swarm Algorithm,” System Engineering Theory and Practice, vol. 22(11), pp.32-38, 2002

[32] S.D. Muller, J. Marchetto, S. Airaghi, P. Kournoutsakos. "Optimization based on bacterial chemotaxis", IEEE Transactions on Evolutionary Computation, 6(1), pp 16-29, 2002 https://doi.org/10.1109/4235.985689.

[33] C. Ferreira. "Gene expression programming in problem solving." In proceedings of Soft computing and industry, pp. 635-653, 2002 https://doi.org/10.1007/978-1-4471-0123-9_54.

[34] Xiao-Feng Xie, Wen-Jun Zhang, Zhi-Lian Yang, "Social cognitive optimization for nonlinear programming problems", Proceedings of the First International Conference on Machine Learning and Cybernetics, Beijing, China, pp 779-783, 2002.

[35] K. M. Passino, -Biomimicry of bacterial foraging for distributed optimization and control, ॥l IEEE Control Syst., vol. 22, no. 3, pp. 52-67, Jun. 2002. https://doi.org/10.1109/MCS.2002.1004010.

[36] M. Eusuff, K.E. Lansey. "Optimization of water distribution network design using the shuffled frog leaping algorithm", Journal of Water Resources Planning and Management, 129(3), pp 210-225, 2003 https://doi.org/10.1061/(ASCE)0733-9496(2003)129:3(210).

[37] B. Webster, P.J. Bernhard. "A local search optimization algorithm based on natural principles of gravitation", In Proceedings of the international conference on information and knowledge engineering (IKE'03), pp 255-261, 2003.

[38] S.H. Jung. "Queen-bee evolution for genetic algorithms", Electronics letters, 39(6), pp 575-576, 2003 https://doi.org/10.1049/el:20030383. 
[39] Ray, Tapabrata, and Kim Meow Liew, "Society and civilization: An optimization algorithm based on the simulation of social behavior", IEEE Transactions on Evolutionary Computation, 7(4), pp 386-396, 2003 https://doi.org/10.1109/TEVC.2003.814902.

[40] X. Li and J. Qian, —Studies on Artificial Fish Swarm Optimization Algorithm based on Decomposition and Coordination Techniques [J], $\|$ J. Circuits Syst., vol. 1, pp. 1-6, 2003.

[41] P. Pinto, T. A. Runkler, J. M. Sousa. "Wasp swarm optimization of logistic systems", Adaptive and Natural Computing Algorithms, pp 264-267, 2005 https://doi.org/10.1007/3-211-27389-1 63.

[42] O. K. Erol, I. Eksin. "A new optimization method: big bang-big crunch", Advances in Engineering Software, 37(2), pp 106-111, 2006 https://doi.org/10.1016/j.advengsoft.2005.04.005.

[43] Shu-Chuan Chu, Pei-Wei Tsai, Jeng-Shyang Pan. "Cat Swarm Optimisation", In Proceedings of the 9th Pacific Rim International Conference on Artificial Intelligence, Guilin, China, pp 854-858, 2006.

[44] N. Hansen, Sibylle, D. Müller, P. Koumoutsakos. "Reducing the Time Complexity of the Derandomized Evolution Strategy with Covariance Matrix Adaptation (CMA-ES)", Evolutionary Computation, 11(1), pp 1-18, 2006 https://doi.org/10.1162/106365603321828970.

[45] A.R. Mehrabian, C. Lucas. "A novel numerical optimization algorithm inspired from weed colonization", Ecological informatics, 1(4), pp 355-366, 2006 https://doi.org/10.1016/j.ecoinf.2006.07.003.

[46] Karci, B. Alatas. "Thinking Capability of Saplings Growing Up Algorithm”, In the proceedings of International Conference on Intelligent Data Engineering and Automated Learning, pp 386-393, 2006 https://doi.org/10.1007/11875581_47.

[47] H Du, X Wu, J Zhuang, "Small-world optimization algorithm for function optimization", Advances in Natural Computation, pp 264-273, 2006 https://doi.org/10.1007/11881223_33.

[48] A.R. Mehrabian and C. Lucas,A novel numerical optimization algorithm inspired from weed colonization,\| Ecol. Inform., vol. 1, no. 4, pp. 355366, 2006 https://doi.org/10.1016/j.ecoinf.2006.07.003.

[49] S. He, Q. H. Wu, and J. R. Saunders, A novel group search optimizer inspired by animal behavioural ecology,\| in Evolutionary Computation, 2006. CEC 2006. IEEE Congress on, 2006, pp. 1272-1278.

[50] D. Karaboga, B. Basturk. "A powerful and efficient algorithm for numerical function optimization: artificial bee colony (ABC) algorithm", Journal of Global Optimization, 39(3) pp 459-471, 2007 https://doi.org/10.1007/s10898-007-9149-x.

[51] R. A. Formato. "Central force optimization: a new metaheuristic with applications in applied electromagnetics", Progress In Electromagnetics Research, 77, 425-491, 2007 https://doi.org/10.2528/PIER07082403.

[52] S. Su, J. Wang, W. Fan, X. Yin. "Good Lattice Swarm Algorithm for Constrained Engineering Design Optimization", In proceedings of the International Conference on Wireless Communications, Networking and Mobile Computing, pp 6421-6424, 2007 https://doi.org/10.1109/WICOM.2007.1575.

[53] E. Atashpaz-Gargari, C. Lucas. "Imperialist competitive algorithm: an algorithm for optimization inspired by imperialistic competition", In Proceedings of the IEEE Congress on Evolutionary Computation, 2007 https://doi.org/10.1109/CEC.2007.4425083.

[54] A.Mucherino, O. Seref. "Monkey search: a novel metaheuristic search for global optimization", Data Mining, Systems Analysis and Optimization in Biomedicine, 953(1), 2007 https://doi.org/10.1063/1.2817338.

[55] Borji, "A new global optimization algorithm inspired by parliamentary political competitions", In Proceedings of the Mexican International Conference on Artificial Intelligence, pp 61-71, 2007 https://doi.org/10.1007/978-3-540-76631-5_7.

[56] P. Rabanal, I. Rodríguez, F. Rubio. "Using river formation dynamics to design heuristic algorithms", In the proceedings of the International Conference on Unconventional Computation, pp 163-177, 2007. https://doi.org/10.1007/978-3-540-73554-0_16.

[57] J. P. Pedroso. "Simple meta-heuristics using the simplex algorithm for non-linear programming”, Technical Report DCC-2007-06, DCC, FC, Universidade do Porto, 2007

[58] Y. Chu, H. Mi, H. Liao. “A Fast Bacterial Swarming Algorithm for high-dimensional function optimization”, In Proceedings of IEEE World Congress on Computational Intelligence, Hong Kong, pp 3135-3140, 2008

[59] D. Simon. "Biogeography-based optimization", IEEE Transactions on Evolutionary Computation, 12(6), pp 702-713, 2008 https://doi.org/10.1109/TEVC.2008.919004.

[60] J. A. B. Filho , F. B. L. Neto, A. J. C. C. Lins, A. I. S. Nascimento, M. P. Lima, "A novel search algorithm based on fish school behavior", In proceedings of IEEE International Conference on Systems, Man and Cybernetics, pp 2646-2651, 2008

[61] W. Cai, W. Yang, X. Chen. "A Global Optimization Algorithm Based on Plant Growth Theory: Plant Growth Optimization", Proceedings of the 2008 International Conference on Intelligent Computation Technology and Automation, pp 1194-1199, 2008 https://doi.org/10.1109/ICICTA.2008.416.

[62] T. C. Havens, C. J. Spain, N. G. Salmon, J. M. Keller. "Roach infestation optimization", In proceedings of the IEEE Swarm Intelligence Symposium, SIS 2008, pp 1-7, 2008 https://doi.org/10.1109/SIS.2008.4668317.

[63] P. Cortés, J. M. García, J. Muñuzuri, L. Onieva. "Viral systems: A new bio-inspired optimisation approach", Computers \& Operations Research, 35(9), pp 2840-2860, 2008 https://doi.org/10.1016/j.cor.2006.12.018.

[64] T. Chen. "A simulative bionic intelligent optimization algorithm: Artificial searching swarm algorithm and its performance analysis". In Proceedings of the IEEE International Joint Conference on Computational Sciences and Optimization, CSO 2009, Vol. 2, pp 864-866, 2009 https://doi.org/10.1109/CSO.2009.183.

[65] S. Das, A. Chowdhury, A. Abraham. "A Bacterial Evolutionary Algorithm for automatic data clustering", In Proceedings of IEEE Congress on Evolutionary Computation, Trondheim, Norway, pp 2403-2410, 2009 https://doi.org/10.1109/CEC.2009.4983241.

[66] S. Iordache. "Consultant-guided search: a new metaheuristic for combinatorial optimization problems", In Proceedings of the 12th annual conference on Genetic and evolutionary computation, Portland, OR, USA, pp. 225-232, 2009 https://doi.org/10.1145/1830483.1830526.

[67] S. Kadioglu, M. Sellmann. "Dialectic Search", In Proceedings of International Conference on Principles and Practice of Constraint Programming, pp 486-500, 2009 https://doi.org/10.1007/978-3-642-04244-7_39.

[68] Y. Shiqin, J. Jianjun, Y. Guangxing. “A Dolphin Partner Optimization”, In proc of 2009 WRI Global Congress on Intelligent Systems, Xiamen, China, pp 124-128, 2009 https://doi.org/10.1109/GCIS.2009.464.

[69] X. Yang. "Firefly algorithms for multimodal optimization." Stochastic algorithms: foundations and applications. Springer Berlin Heidelberg, pp 169-178, 2009 https://doi.org/10.1007/978-3-642-04944-6 14.

[70] K. N. Krishnanand, D. Ghose. "Glowworm swarm optimization for simultaneous capture of multiple local optima of multimodal functions", Swarm intelligence, 3(2), pp 87-124, 2009 https://doi.org/10.1007/s11721-008-0021-5.

[71] S. He, Q.H. Wu, J.R. Saunders. "Group search optimizer: an optimization algorithm inspired by animal searching behavior", IEEE Transactions on evolutionary computation, 13(5), pp 973-990, 2009 https://doi.org/10.1109/TEVC.2009.2011992.

[72] L.M. Zhang, C. Dahlmann, Y. Zhang. "Human-inspired algorithms for continuous function optimization", In proceeding of the IEEE International Conference on Intelligent Computing and Intelligent Systems, ICIS 2009, Vol. 1, pp 318-321, 2009 https://doi.org/10.1109/ICICISYS.2009.5357838.

[73] R. Oftadeh, M. J. Mahjoob. "A new meta-heuristic optimization algorithm: Hunting Search", In proceeding of the Fifth International Conference on Soft Computing, Computing with Words and Perceptions in System Analysis, Decision and Control, 2009 https://doi.org/10.1109/ICSCCW.2009.5379451.

[74] H. Shah-Hosseini. "The intelligent water drops algorithm: a nature-inspired swarm-based optimization algorithm", International Journal of BioInspired Computation, 1(1/2), pp 71-79, 2009 https://doi.org/10.1504/IJBIC.2009.022775.

[75] A.H. Kashan. "League Championship Algorithm: A New Algorithm for Numerical Function Optimization", In proceedings of International Conference of Soft Computing and Pattern Recognition, Malacca, Malaysia, pp 43-48, 2009 https://doi.org/10.1109/SoCPaR.2009.21. 
[76] S. Chen. "Locust Swarms - A new multi-optima search technique", In proceeding of the IEEE Congress on Evolutionary Computation, Trondheim, Norway, pp 1745-1752, 2009 https://doi.org/10.1109/CEC.2009.4983152.

[77] U. Premaratne, J. Samarabandu, T. Sidhu. "A new biologically inspired optimization algorithm" In proceedings of the 2009 international conference on industrial and information systems, pp 279-284, 2009 https://doi.org/10.1109/ICIINFS.2009.5429852.

[78] Y. Marinakis, M. Marinaki, and N. Matsatsinis, -A hybrid bumble bees mating optimization-grasp algorithm for clustering,l in Hybrid Artificial Intelligence Systems, Springer, 2009, pp. 549-556. https://doi.org/10.1007/978-3-642-02319-4 66.

[79] R. Oftadeh and M. J. Mahjoob, - A new meta-heuristic optimization algorithm: Hunting Search, $\|$ in Soft Computing, Computing with Words and Perceptions in System Analysis, Decision and Control, 2009. ICSCCW 2009. Fifth International Conference on, 2009 , pp. 1-5. https://doi.org/10.1109/ICSCCW.2009.5379451.

[80] Xin-She Yang. "A new metaheuristic bat-inspired algorithm", In Proceedings of the Fourth International Workshop on Nature inspired cooperative strategies for optimization (NICSO 2010), Berlin, Heidelberg, pp 65-74, 2010 https://doi.org/10.1007/978-3-642-12538-6_6

[81] A.Kaveh, S. Talatahari. "A novel heuristic optimization method: charged system search", Acta Mechanica, 213(3-4), pp 267-289, 2010 https://doi.org/10.1007/s00707-009-0270-4.

[82] X.S. Yang, S. Deb. "Eagle strategy using Lévy walk and firefly algorithms for stochastic optimization." In Proceedings of Nature Inspired Cooperative Strategies for Optimization (NICSO 2010), pp. 101-111, 2010 https://doi.org/10.1007/978-3-642-12538-6_9.

[83] Y. Tan, Y. Zhu. "Fireworks algorithm for optimization"”, In proceedings of International Conference in Swarm Intelligence, pp 355-364, 2010 https://doi.org/10.1007/978-3-642-13495-1_44.

[84] A.Ahrari, A. A. Atai. "Grenade explosion method - a novel tool for optimization of multimodal functions", Applied Soft Computing, 10(4), pp 1132-1140, 2010 https://doi.org/10.1016/j.asoc.2009.11.032.

[85] M.A. Eita, M. M. Fahm. "Group counseling optimization: a novel approach", In proceedings of Research and Development in Intelligent Systems XXVI, pp 195-208, 2010 https://doi.org/10.1007/978-1-84882-983-1_14.

[86] H. Chen, Y. Zhu, K. Hu, X. He. "Hierarchical Swarm Model: A New Approach to Optimization”, Discrete Dynamics in Nature and Society, 2010 https://doi.org/10.1155/2010/379649.

[87] A.Sharma. "A new optimizing algorithm using reincarnation concept", In the proceeding of the 11th IEEE International Symposium on Computational Intelligence and Informatics (CINTI), pp. 281-288, 2010 https://doi.org/10.1109/CINTI.2010.5672231.

[88] Y. Xu, Z. Cui, J. Zeng, "Social emotional optimization algorithm for nonlinear constrained optimization problems." In Proceedings of the International Conference on Swarm, Evolutionary, and Memetic Computing, pp 583-590, 2010 https://doi.org/10.1007/978-3-642-17563-3 68.

[89] R. Hedayatzadeh, F. A. Salmassi, R. Akbari, K. Ziarati. "Termite colony optimization: A novel approach for optimizing continuous problems", In the proceedings of 2010 18th IEEE Iranian Conference on Electrical Engineering, pp. 553-558, 2010 https://doi.org/10.1109/IRANIANCEE.2010.5507009.

[90] Z. Bayraktar, M. Komurcu, D. H. Werner. "Wind Driven Optimization (WDO): A novel nature-inspired optimization algorithm and its application to electromagnetics", In proceedings of 2010 IEEE Antennas and Propagation Society International Symposium, pp 1-4, 2012 https://doi.org/10.1109/APS.2010.5562213.

[91] X.-S. Yang, —A New Metaheuristic Bat-Inspired Algorithm,\| in Nature Inspired Cooperative Strategies for Optimization (NICSO 2010), J. R. González, D. A. Pelta, C. Cruz, G. Terrazas, and N. Krasnogor, Eds. Springer Berlin Heidelberg, 2010, pp. 65-74. https://doi.org/10.1007/978-3642-12538-6_6.

[92] Alatas. “ACROA: Artificial Chemical Reaction Optimization Algorithm for global optimization”, Expert Systems with Applications, 38(10), pp 13170-13180, $2011 \mathrm{https} / / /$ doi.org/10.1016/j.eswa.2011.04.126.

[93] R.S. Parpinelli, H.S. Lopes. "An eco-inspired evolutionary algorithm applied to numerical optimization." In proc. of the Third World Congress on Nature and Biologically Inspired Computing (NaBIC), 2011, Spain, pp 466-471, 2011 https://doi.org/10.1109/NaBIC.2011.6089631.

[94] H. Shah-Hosseini. "Principal components analysis by the galaxy-based search algorithm: a novel metaheuristic for continuous optimisation", International Journal of Computational Science and Engineering, 6(1/2), pp 132-140, 2011 https://doi.org/10.1504/IJCSE.2011.041221.

[95] Duman, M. Uysal, A. F. Alkaya1. "Migrating Birds Optimization: A New Meta-heuristic Approach and Its Application to the Quadratic Assignment Problem", In proceedings of the European Conference on the Applications of Evolutionary Computation, pp 254-263, 2011 https://doi.org/10.1007/978-3-642-20525-5 26.

[96] A.Salhi, E. S. Fraga. "Nature-Inspired Optimisation Approaches and the New Plant Propagation Algorithm", In Proceedings of the The International Conference on Numerical Analysis and Optimization (ICeMATH '11), Yogyakarta, Indonesia, pp K2-1-K2-8, 2011

[97] K. Tamura, K. Yasuda, "Spiral Dynamics Inspired Optimization." Journal of Advanced Computational Intelligence and Intelligent Informatics, 15(8), pp 1116-1122, 2011 https://doi.org/10.20965/jaciii.2011.p1116.

[98] R. V. Rao, V. J. Savsani, D. P. Vakharia. "Teaching-learning-based optimization: a novel method for constrained mechanical design optimization problems", Computer-Aided Design, 43, (3), pp 303-315, 2011 https://doi.org/10.1016/j.cad.2010.12.015.

[99] T. H. Tran, K. M. Ng. "A water-flow algorithm for flexible flow shop scheduling with intermediate buffers", Journal of Scheduling, 14(5), pp 483500, 2011 https://doi.org/10.1007/s10951-010-0205-x.

[100] H. Shayeghi, J. Dadashpour. "Anarchic Society Optimization Based PID Control of an Automatic Voltage Regulator (AVR) System", Electrical and Electronic Engineering, 2(4),pp. 199-207, 2012 https://doi.org/10.5923/j.eee.20120204.05.

[101] J. Li, Z. Cui, Z. Shi. "An improved artificial plant optimization algorithm for coverage problem in WSN", Sensor Letters, 10(8), pp 1874-1878, 2012 https://doi.org/10.1166/s1.2012.2627.

[102] B.Niu, H. Wang. "Bacterial Colony Optimization”, Discrete Dynamics in Nature and Society, 2012 https://doi.org/10.1155/2012/698057.

[103] A.Milani, V. Santucci. "Community of scientist optimization: An autonomy oriented approach to distributed optimization", AI Communications, 25(2), pp. 157-172, 2012

[104] P. Civicioglu. "Transforming geocentric cartesian coordinates to geodetic coordinates by using differential search algorithm", Computers \& Geosciences, 46, pp 229-247, 2012 https://doi.org/10.1016/j.cageo.2011.12.011.

[105] Cuevas, D. Oliva, D. Zaldivar, M. Pérez-Cisneros, H. Sossa. "Circle detection using electro-magnetism optimization", Information Sciences, 182(1), pp 40-55, 2012 https://doi.org/10.1016/j.ins.2010.12.024.

[106] X. Yang. "Flower pollination algorithm for global optimization." In Proceedings of International Conference on Unconventional Computing and Natural Computation, pp 240-249, 2012 https://doi.org/10.1007/978-3-642-32894-7 27.

[107] W. T. Pan. "A new fruit fly optimization algorithm: taking the financial distress model as an example", Knowledge-Based Systems, 26, pp 6974, 2012 https://doi.org/10.1016/j.knosys.2011.07.001.

[108] A.Mozaffari, A. Fathi, S. Behzadipour. "The great salmon run: a novel bio-inspired algorithm for artificial system design and optimisation", International Journal of Bio-Inspired Computation, 4(5), pp 286-301, 2012 https://doi.org/10.1504/IJBIC.2012.049889.

[109] M. El-Dosuky, A. El-Bassiouny, T. Hamza, M. Rashad. "New hoopoe heuristic optimization", International Journal of Science and Advanced Technology, 2(9), pp 85-90, 2012

[110] H. Hernández, C. Blum. "Distributed graph coloring: an approach based on the calling behavior of Japanese tree frogs", Swarm Intelligence, 6(2), pp 117-150 https://doi.org/10.1007/s11721-012-0067-2.

[111] A.H. Gandomi, A. H. Alavi. "Krill herd: a new bio-inspired optimization algorithm ", Communications in Nonlinear Science and Numerical Simulation, 17(12), pp 4831-4845, 2012 https://doi.org/10.1016/j.cnsns.2012.05.010.

[112] Sadollah, A. Bahreininejad, H. Eskandar, M. Hamdi. "Mine blast algorithm for optimization of truss structures with discrete variables", Computers and Structures, (102-103), pp 49-63, 2012 https://doi.org/10.1016/j.compstruc.2012.03.013.

[113] Kaveh, M. Khayatazad. "A new meta-heuristic method: ray optimization", Computers \& Structures, (112), pp 283-294, 2012 https://doi.org/10.1016/j.compstruc.2012.09.003. 
[114] H.D. Purnomo, H.-M. Wee., "Soccer game optimization: an innovative integration of evolutionary algorithm and swarm intelligence algorithm", Meta-Heuristics optimization algorithms in engineering, business, economics, and finance. IGI Global, 2012

[115] H. Eskandar, A. Sadollah, A. Bahreininejad, M. Hamdi. "Water cycle algorithm - A novel metaheuristic optimization method for solving constrained engineering optimization problems", Computers \& Structures, 110-111, pp 151-166, 2012 https://doi.org/10.1016/j.compstruc.2012.07.010.

[116] R. Tang, S. Fong, X. S. Yang, S. Deb. "Wolf search algorithm with ephemeral memory". In proceedings of Seventh International Conference on Digital Information Management, pp 165-172, 2012 https://doi.org/10.1109/ICDIM.2012.6360147.

[117] H. T. Nguyen, B. Bhanu. "Zombie Survival Optimization: A swarm intelligence algorithm inspired by zombie foraging", In Proceedings of 21st IEEE International Conference on Pattern Recognition (ICPR), Tsukuba, Japan, pp 987-990, 2012

[118] Cuevas, M. González, D. Zaldivar, M. Pérez-Cisneros, and G. García, -An algorithm for global optimization inspired by collective animal behavior, \|l Discrete Dyn. Nat. Soc., vol. 2012, 2012. https://doi.org/10.1155/2012/638275.

[119] A.Kaveh, N. Farhoudi. "A new optimization method: Dolphin echolocation”, Advances in Engineering Software, 59, pp.53-70, 2013 https://doi.org/10.1016/j.advengsoft.2013.03.004.

[120] A.Subramanian, A. S. S. Sekar, K. Subramanian. "A New Engineering Optimization Method: African Wild Dog Algorithm", International Journal of Soft Computing, 8(3), pp 163-170, 2013

[121] W. Yan, Z. J. Hao. "A novel optimization algorithm based on atmosphere clouds model", International Journal of Computational Intelligence and Applications, 12(01), p.1350002, 2013 https://doi.org/10.1142/S1469026813500028.

[122] P. Civicioglu. "Backtracking Search Optimization Algorithm for numerical optimization problems", Applied Mathematics and Computation, 29(15), pp. 8121-8144, 2013 https://doi.org/10.1016/j.amc.2013.02.017.

[123] A.Hatamlou. "Black hole: A new heuristic optimization approach for data clustering", Information Sciences, 222, pp 175-184, 2013 https://doi.org/10.1016/j.ins.2012.08.023.

[124] M. Taherdangkoo, M. H. Shirzadi M. Yazdi, M. H. Bagheri. "A robust clustering method based on blind, naked mole-rats (BNMR) algorithm", Swarm and Evolutionary Computation, 10, pp 1-11, 2013 https://doi.org/10.1016/j.swevo.2013.01.001.

[125] A.S. Eesa, A.M. Abdulazeez, Z. Orman. "Cuttlefish algorithm - a novel bio-inspired optimization algorithm", International Journal of Scientific and Engineering Research, 4(9), pp. 1978-1986, 2013 https://doi.org/10.1007/978-3-642-37371-8_26.

[126] Sur, S. Sharma, A. Shukla. "Egyptian vulture optimization algorithm - a new nature inspired meta-heuristics for knapsack problem", In proceedings of the 9th International Conference on Computing and Information Technology (IC2IT), Bangkok, pp. 227-237, 2013

[127] M. Abdechiri, M.R. Meybodi, H. Bahrami. "Gases Brownian motion optimization: an algorithm for optimization (GBMO)", Applied Soft Computing, 13(5), pp 2932-2946, 2013 https://doi.org/10.1016/j.asoc.2012.03.068.

[128] Mo, L. Xu. "Magnetotactic bacteria optimization algorithm for multimodal optimization", In the proceedings of the 2013 IEEE Symposium on Swarm Intelligence (SIS), pp 240-247, 2013 https://doi.org/10.1109/SIS.2013.6615185.

[129] Y. Gheraibia, A. Moussaoui. "Penguins search optimization algorithm (PeSOA)", In proceedings of the International Conference on Industrial, Engineering and Other Applications of Applied Intelligent Systems, pp 222-231, 2013 https://doi.org/10.1007/978-3-642-38577-3 23.

[130] Erik Cuevas,Miguel Cienfuegos, Daniel Zaldívar , Marco Pérez-Cisneros, "A swarm optimization algorithm inspired in the behavior of the social-spider", Expert Systems with Applications, 40(16), pp 6374-6384, 2013 https://doi.org/10.1016/j.eswa.2013.05.041.

[131] M. Neshat, G. Sepidnam, M. Sargolzaei, "Swallow swarm optimization algorithm: a new method to optimization", Neural Computing and Applications, 23(2), pp 429-454, 2013 https://doi.org/10.1007/s00521-012-0939-9.

[132] X. X. Li, J. Zhang, M. Yin. "Animal migration optimization: an optimization algorithm inspired by animal migration behavior", Neural Computing and Applications, 24(7), pp 1867-1877, 2014 https://doi.org/10.1007/s00521-013-1433-8.

[133] M. T. Adham, P. J. Bentley. "An Artificial Ecosystem Algorithm applied to static and Dynamic Travelling Salesman Problems", In Proceedings of the IEEE International Conference on Evolvable Systems, Orlando, FL, USA, pp 149-156, 2014 https://doi.org/10.1109/ICES.2014.7008734.

[134] A.Askarzadeh. "Bird mating optimizer: an optimization algorithm inspired by bird mating strategies", Communications in Nonlinear Science and Numerical Simulation, 19(4), pp1213-1228, 2014 https://doi.org/10.1016/j.cnsns.2013.08.027.

[135] X. Meng, Y. Liu, X. Gao, H. Zhang. “A New Bio-inspired Algorithm: Chicken Swarm Optimization”, In Proceedings of ICSI 2014, vol 8794, pp 86-94, 2014 https://doi.org/10.1007/978-3-319-11857-4 10.

[136] C. Obagbuwa, A. O. Adewumi. "An Improved Cockroach Swarm Optimization", The Scientific World Journal, 2014 https://doi.org/10.1155/2014/375358.

[137] A.Kaveh, V. R. Mahdavi. "Colliding bodies optimization: a novel meta-heuristic method", Computers \& Structures, 139, pp 18-27, 2014 https://doi.org/10.1016/j.compstruc.2014.04.005.

[138] S. Salcedo-Sanz, J. Del Ser, I. Landa-Torres, S. Gil-López, J. A. Portilla-Figueras. "The coral reefs optimization algorithm: a novel metaheuristic for efficiently solving optimization problems", The Scientific World Journal, 2014 https://doi.org/10.1155/2014/739768.

[139] N. Ghorbani, E. Babaei. "Exchange market algorithm." Applied Soft Computing, 19, pp 177-187, 2014 https://doi.org/10.1016/j.asoc.2014.02.006.

[140] M. Ghaemi, M. R. F. Derakhshi. "Forest Optimization Algorithm",_Expert Systems with Applications, 41(15), 6676-6687, 2014 https://doi.org/10.1016/j.eswa.2014.05.009.

[141] E. Osaba, F. Diaz, E. Onieva. "Golden ball: a novel meta-heuristic to solve combinatorial optimization problems based on soccer concepts", Applied Intelligence. 41(1), pp 145-166, 2014 https://doi.org/10.1007/s10489-013-0512-y.

[142] J.M.L. Melvix. "Greedy Politics Optimization: Metaheuristic inspired by political strategies adopted during state assembly elections", In proceedings of the IEEE International Advance Computing Conference (IACC), pp 1157-1162, 2014

[143] S. Mirjalili, S. M. Mirjalili, A. Lewis. "Grey wolf optimizer." Advances in Engineering Software, 69, pp 46-61, 2014 https://doi.org/10.1016/j.advengsoft.2013.12.007.

[144] A.Hatamlou. "Heart: a novel optimization algorithm for cluster analysis", Progress in Artificial Intelligence, 2(2), pp 167-173, 2014 https://doi.org/10.1007/s13748-014-0046-5.

[145] H. Gandomi. "Interior search algorithm (ISA): a novel approach for global optimization", ISA transactions, 53(4), pp 1168-1183, 2014 https://doi.org/10.1016/j.isatra.2014.03.018.

[146] V. V. Melo. "Kaizen Programming", In Proceedings of the 2014 Annual Conference on Genetic and Evolutionary Computation (GECCO), pp 895-902, 2014 https://doi.org/10.1145/2576768.2598264.

[147] M. Hajiaghaei-Keshteli, M. Aminnayeri. "Solving the integrated scheduling of production rail transportation problem by Keshtel algorithm", Applied Soft Computing, 25, pp 184-203, 2014 https://doi.org/10.1016/j.asoc.2014.09.034.

[148] A.Brabazon, W. Cui, M. O’Neill. “The raven roosting optimisation algorithm”, Soft Computing, 20(2), pp 525-545, 2014 https://doi.org/10.1007/s00500-014-1520-5.

[149] Felipe, E. Goldbarg, M. Goldbarg. "Scientific algorithms for the Car Renter Salesman Problem." In Proceedings of the IEEE Congress on Evolutionary Computation (CEC), Beijing, China, pp. 873-879, 2014 https://doi.org/10.1109/CEC.2014.6900556.

[150] O. Abedinia, N. Amjady, A. Ghasemi. "A new metaheuristic algorithm based on shark smell optimization", Complexity, 2014 https://doi.org/10.1002/cplx.21634.

[151] Jagdish Chand Bansal, Harish Sharma, Shimpi Singh Jadon, Maurice Clerc, "Spider monkey optimization algorithm for numerical optimization", Memetic Computing, 6(1), pp 31-47, 2014 https://doi.org/10.1007/s12293-013-0128-0.

[152] F. Merrikh-Bayat, "A Numerical Optimization Algorithm Inspired by the Strawberry Plant', arXiv preprint arXiv:1407.7399, 2014

[153] M.Y. Cheng, D. Prayogo. "Symbiotic organisms search: a new metaheuristic optimization algorithm”, Computers \& Structures, 139, pp 98-112, 2014 https://doi.org/10.1016/j.compstruc.2014.03.007. 
[154] J.P. Arnaout. "Worm Optimization: A novel optimization algorithm inspired by C. Elegans". In Proceedings of the 2014 International Conference on Industrial Engineering and Operations Management, pp 2499-2505, 2014

[155] S. Mirjalili. "The ant lion optimizer", Advances in Engineering Software, 83, pp 80-98, 2015 https://doi.org/10.1016/j.advengsoft.2015.01.010.

[156] S. U. Ali, G. Tezel, E. Yel. “Artificial algae algorithm (AAA) for nonlinear global optimization”, Applied Soft Computing, 31, pp 153-157, 2013 https://doi.org/10.1016/j.asoc.2015.03.003.

[157] Y. Shi. "An optimization algorithm based on brainstorming process", Emerging Research on Swarm Intelligence and Algorithm Optimization, pp 1-35, 2015 https://doi.org/10.4018/978-1-4666-6328-2.ch001.

[158] Oguz FINDIK. "Bull optimization algorithm based on genetic operators for continuous optimization problems", Turkish Journal of Electrical Engineering \& Computer Sciences, 23, pp 2225-2239, 2015 https://doi.org/10.3906/elk-1307-123.

[159] G. Wang, S. Deb, L. S. Coelho, "Elephant Herding Optimization", In proc of the 3rd International Symposium on Computational and Business Intelligence (ISCBI), Bali, Indonesia, pp 1-5, 2015 https://doi.org/10.1109/ISCBI.2015.8.

[160] S. Deb, S. Fong, Z. Tian. "Elephant Search Algorithm for optimization problems", In Proc. of the 10th IEEE International Conference on Digital Information Management (ICDIM), pp 249-255, 2015 https://doi.org/10.1109/ICDIM.2015.7381893.

[161] H. Beiranvand, E. Rokrok. "General Relativity Search Algorithm: A Global Optimization Approach", International Journal of Computational Intelligence and Applications, 14(3), 2015 https://doi.org/10.1142/S1469026815500170.

[162] Tang, S. Dong, Y. Jiang, H. Li, Y. Huang. "ITGO: Invasive tumor growth optimization algorithm", Applied Soft Computing, (36), pp. 670-698, 2015 https://doi.org/10.1016/j.asoc.2015.07.045.

[163] Javidy, A. Hatamlou, S. Mirjalili. "Ions motion algorithm for solving optimization problems", Applied Soft Computing, 32(1), pp 72-79, 2015 https://doi.org/10.1016/j.asoc.2015.03.035.

[164] Chen, Y. Tsai, I. Liu, C. Lai, Y. Yeh, S. Kuo, Y. Chou. "A Novel Metaheuristic: Jaguar Algorithm with Learning Behavior." In Proceedings of the IEEE International Conference on Systems, Man, and Cybernetics (SMC), pp. 1595-1600, 2015 https://doi.org/10.1109/SMC.2015.282.

[165] H. Shareef, A.A. Ibrahim, A.H. Mutlag. "Lightning search algorithm", Applied Soft Computing, 36(1), pp 315-333, 2015 https://doi.org/10.1016/i.asoc.2015.07.028.

[166] G. Wang, S. Deb, Z. Cui. "Monarch butterfly optimization",, Neural Computing and Applications, pp 1-20, 2015.

[167] S. Mirjalili. "Moth-flame optimization algorithm: A novel nature-inspired heuristic paradigm", Knowledge-Based Systems, 89, 228-249, 2015 https://doi.org/10.1016/j.knosys.2015.07.006.

[168] S. Mirjalili, S. M. Mirjalili, A. Hatamlou. "Multi-Verse Optimizer: a nature-inspired algorithm for global optimization." Neural Computing \& Applications, 27(2), pp 1-19, 2015 https://doi.org/10.1007/s00521-015-1870-7.

[169] A.H. Kashan. "A new metaheuristic for optimization: optics inspired optimization(OIO)", Computers \& Operations Research, 55, pp.99-125, 2015 https://doi.org/10.1016/j.cor.2014.10.011.

[170] X. Hea, S. Zhang, J. Wang. "A Novel Algorithm Inspired by Plant Root Growth with Self-similarity Propagation", In proceedings of the 1st International Conference on Industrial Networks and Intelligent Systems (INISCom), pp 157-162, 2015 https://doi.org/10.4108/icst.iniscom.2015.258407.

[171] Merrikh-Bayat. "The runner-root algorithm: A metaheuristic for solving unimodal and multimodal optimization problems inspired by runners and roots of plants in nature", Applied Soft Computing, (33), pp 292-303, 2015 https://doi.org/10.1016/j.asoc.2015.04.048.

[172] H. Salimi, "Stochastic fractal search: a powerful metaheuristic algorithm", Knowledge-Based Systems, 75, pp1-18, 2015 https://doi.org/10.1016/j.knosys.2014.07.025.

[173] Dogan, T. Olmez. "A new metaheuristic for numerical function optimization: Vortex Search Algorithm", Information Sciences, 293, pp 125145, 2015 https://doi.org/10.1016/j.ins.2014.08.053.

[174] Y. J. Zheng. "Water wave optimization: a new nature-inspired metaheuristic", Computers \& Operations Research, 55, pp 1-11, 2015 https://doi.org/10.1016/i.cor.2014.10.008

[175] B. Odili, M. N. M. Kahar. "Solving the Traveling Salesman's Problem Using the African Buffalo Optimization". Computational intelligence and neuroscience, vol. 2016, Article ID 1510256, 12 pages, 2016 https://doi.org/10.1155/2016/1510256.

[176] Xian-Bing Meng, X.Z. Gao, Lihua Lu, Yu Liu \& Hengzhen Zhang. "A new bio-inspired optimisation algorithm: Bird Swarm Algorithm", Journal of Experimental \& Theoretical Artificial Intelligence, 28(4), pp 673-687, 2016 https://doi.org/10.1080/0952813X.2015.1042530.

[177] M. K. Ibrahim, R. S. Ali. "Novel Optimization Algorithm Inspired by Camel Traveling Behavior", Iraq J. Electrical and Electronic Engineering, 12(2), 167-178, 2016 https://doi.org/10.33762/eeej.2016.118375.

[178] X. Feng, M. Ma, H. Yu. "Crystal Energy Optimization Algorithm", Computational Intelligence, 32(2), pp 284-322, 2016 https://doi.org/10.1111/coin.12053.

[179] S. Mirjalili. "Dragonfly algorithm: a new meta-heuristic optimization technique for solving single-objective, discrete, and multi-objective problems", Neural Computing and Applications, 27(4), pp 1053-1073, 2016 https://doi.org/10.1007/s00521-015-1920-1.

[180] N. Razmjooy, M. Khalilpour, M. Ramezani. "A New Meta-Heuristic Optimization Algorithm Inspired by FIFA World Cup Competitions: Theory and Its Application in PID Designing for AVR System", Journal of Control, Automation and Electrical Systems, 27(4), 1-22, 2016 https://doi.org/10.1007/s40313-016-0242-6.

[181] A.E. Xavier, V. L. Xavier. "Flying elephants: a general method for solving non-differentiable problems", Journal of Heuristics, 22(4), pp 649664, 2016 https://doi.org/10.1007/s10732-014-9268-8.

[182] M. Yazdani, F. Jolai. "Lion Optimization Algorithm (LOA): A nature-inspired metaheuristic algorithm", Journal of Computational Design and Engineering, 3(1), pp 24-36, 2016 https://doi.org/10.1016/j.jcde.2015.06.003.

[183] Y. Labbi, D. B. Attous, H. A. Gabbar, B. Mahdad, A. Zidan. "A new rooted tree optimization algorithm for economic dispatch with valvepoint effect”, International Journal of Electrical Power \& Energy Systems, 79, pp 298-311, 2016 https://doi.org/10.1016/j.ijepes.2016.01.028.

[184] A.Ebrahimi, E. Khamehchi, "Sperm Whale Algorithm: an Effective Metaheuristic Algorithm for Production Optimization Problems", Journal of Natural Gas Science \& Engineering, 29, pp 211-222, 2016 https://doi.org/10.1016/j.jngse.2016.01.001.

[185] M. D. Li, H. Zhao, X. W. Weng, T. Han. "A novel nature-inspired algorithm for optimization: Virus colony search", Advances in Engineering Software, 92, pp 65-88, 2016 https://doi.org/10.1016/j.advengsoft.2015.11.004.

[186] Y. C. Liang, J. R. C. Juarez. "A novel metaheuristic for continuous optimization problems: Virus optimization algorithm”, Engineering Optimization, 48(1), pp 73-93, 2016 https://doi.org/10.1080/0305215X.2014.994868.

[187] A.Kaveh, T. Bakhshpoori. "Water Evaporation Optimization: A novel physically inspired optimization algorithm", Computers \& Structures, 167, pp 69-85, 2016 https://doi.org/10.1016/j.compstruc.2016.01.008.

[188] S. Mirjalili, A. Lewisa. "The Whale Optimization Algorithm", Advances in Engineering Software, 95, pp 51-67, 2016 https://doi.org/10.1016/j.advengsoft.2016.01.008.

[189] S. Saremi, S. Mirjalili, A. Lewis. "Grasshopper Optimisation Algorithm: Theory and application",__Advances in Engineering Software, 105, pp 30-47, 2017 https://doi.org/10.1016/j.advengsoft.2017.01.004.

[190] Raouf, Hezam, "Sperm motility algorithm: a novel metaheuristic approach for global optimisation", International Journal of Operational Research (IJOR), 28(2), 2017 https://doi.org/10.1504/IJOR.2017.10002079.

[191] H. Shayanfar and F. Gharehchopogh, "Farmland fertility: A new metaheuristic algorithm for solving continuous optimization problems", Applied Soft Computing, vol. 71, pp. 728-746, 2018. Available: 10.1016/j.asoc.2018.07.033. https://doi.org/10.1016/j.asoc.2018.07.033.

[192] S. Shadravan, H. Naji and V. Bardsiri, "The Sailfish Optimizer: A novel nature-inspired metaheuristic algorithm for solving constrained engineering optimization problems", Engineering Applications of Artificial Intelligence, vol. 80, pp. 20-34, 2019. Available: 10.1016/j.engappai.2019.01.001. https://doi.org/10.1016/j.engappai.2019.01.001. 
[193] H. Wang et al., "Heterogeneous pigeon-inspired optimization", Science China Information Sciences, vol. 62, no. 7, 2019. Available: 10.1007/s11432-018-9713-7. https://doi.org/10.1007/s11432-018-9713-7.

[194] R. Asaad and N. Abdulnabi, "Using Local Searches Algorithms with Ant Colony Optimization for the Solution of TSP Problems", Academic Journal of Nawroz University, vol. 7, no. 3, pp. 1-6, 2018. https://doi.org/10.25007/ajnu.v7n3a193.

[195] A. Heidari, S. Mirjalili, H. Faris, I. Aljarah, M. Mafarja and H. Chen, "Harris hawks optimization: Algorithm and applications", Future Generation Computer Systems, vol. 97, pp. 849-872, 2019. Available: 10.1016/j.future.2019.02.028. https://doi.org/10.1016/j.future.2019.02.028.

[196] T. Biyanto et al., "Optimization of Energy Efficiency and Conservation in Green Building Design Using Duelist, Killer-Whale and Rain-Water Algorithms", IOP Conference Series: Materials Science and Engineering, vol. 267, p. 012036, 2017. Available: 10.1088/1757-899x/267/1/012036 [Accessed 1 November 2019]. https://doi.org/10.1088/1757-899X/267/1/012036.

[197] W. Ahmad, W. Jacqueline; N. Ajit (2017). "Hydrological Cycle Algorithm for Continuous Optimization Problems". Journal of Optimization. 2017: 1-25. https://doi.org/10.1155/2017/3828420.

[198] Harifi, Sasan; Khalilian, Madjid; Mohammadzadeh, Javad; Ebrahimnejad, Sadoullah (2019). "Emperor Penguins Colony: A new metaheuristic algorithm for optimization". Evolutionary Intelligence. 12 (2): 211-226. https://doi.org/10.1007/s12065-019-00212-x.

[199] R. Balamurugan; A.M. Natarajan; K. Premalatha (2015). "Stellar-Mass Black Hole Optimization for Biclustering Microarray Gene Expression Data". Applied Artificial Intelligence an International Journal. 29 (4): 353-381. https://doi.org/10.1080/08839514.2015.1016391.

[200] Metaheuristic | Wikiwand", Wikiwand, $2019 . \quad$ Available: https://www.wikiwand.com/en/Metaheuristic\#/citenoterobbins52stochastic21. [Accessed: 02- Nov- 2019].

[201] S. Almufti, R. Marqas, and V. Ashqi, (2019). Taxonomy of bio-inspired optimization algorithms. Journal of Advanced Computer Science \& Technology, 8(2), 23. https://doi.org/10.14419/jacst.v8i2.29402.

[202] S. Almufti, R. Marqas, and R. Asaad, (2019). Comparative study between elephant herding optimization (EHO) and U-turning ant colony optimization (U-TACO) in solving symmetric traveling salesman problem (STSP). Journal of Advanced Computer Science \& Technology, 8(2), 32. https://doi.org/10.14419/jacst.v8i2.29403.

[203] S. Almufti, A. Zebari, and H. Omer, (2019). A comparative study of particle swarm optimization and genetic algorithm. Journal of Advanced Computer Science \& Technology, 8(2), 40-45. https://doi.org/10.14419/jacst.v8i2.29402. 